\title{
Vergleich von Implementierungsmöglichkeiten industrieller Energiemonitoringsysteme für KMU
}

\author{
René von Lipinski*, Dirk Buße, Jörg Reiff-Stephan
}

\section{Zusammenfassung}

Um den Herausforderungen zukünftiger Wertschöpfungsnetzwerke gewachsen zu sein, sind produzierende Unternehmen im verstärkten Maße auf die Erfassung energetischer Daten angewiesen. Somit gewinnen industrielle Energiemonitoringsysteme zunehmend an Bedeutung. Bei der Erweiterung bestehender Strukturen um die Funktionalität des Energiemonitorings können verschieden konzeptionelle Ansätze verfolgt werden. Diese werden in der vorliegenden Arbeit aufgezeigt und anhand exemplarischer Versuchsaufbauten nutzwertanalytisch verglichen. Es ist festzustellen, dass die Auswahl eines geeigneten Systems für jeden Anwendungsfall individuell zu betrachten ist. Nur unter Berücksichtigung der technischen sowie menschlichen Entitäten innerhalb eines Unternehmens und genauer Definition der Spezifikationen kann aus der breiten Auswahl marktverfügbarer Systeme die jeweils geeignetste Lösung ausgewählt werden.

\begin{abstract}
To meet the challenges of future value-added networks, production companies are dependent on a large-scale capture of energy data. Therefore, industrial energy monitoring systems are becoming increasingly important. For the expansion of existing structures to the functionality of the energy monitoring, different conceptual approaches can be pursued. These are shown in the present research publication, and compared analytically by utility-based exemplary experimental setups. As a result of this investigation, it can be stated that the selection of a suitable system for each application must be considered individually. The selection of an appropriate implementation of an energy monitoring system is dependent on certain factors. These are the technical and human entities within a company, as well as the exact definition of the specifications.
\end{abstract}

\section{Einleitung}

Die informationstechnische Vernetzung industrieller Produktionsprozesse schreitet weiter voran, wobei die Zukunftsinitiative Industrie 4.0 als Katalysator fungiert (Krüger et al. 2015). Die Vorzüge, welche aus derartigen Umstrukturierungen resultieren, sind vielschichtig und sollen dabei helfen, Unternehmen wie auch den Wirtschaftsstandort Deutschland langfristig zu sichern (Bauer et al. 2013). Ein wichtiger Aspekt hierbei ist der effiziente Umgang mit begrenzten Ressourcen (Dorst et al. 2015). Infolgedessen gewinnt auch das intelligente Management energetischer Ressourcen innerhalb der neuen Wertschöpfungsnetzwerke zunehmend an Bedeutung. Dementsprechend bietet der Markt aktuell eine Vielzahl von Produkten für die Realisierung eines Energiemonitoringsystems. Doch gerade kleine und mittlere Unternehmen (KMU) Unternehmen stehen häufig vor der Herausforderung, dass für die Investition in neue Technologien nur ein begrenzter Finanzierungsrahmen zur Verfügung steht. Somit ist es erforderlich, dass sich derartige Systeme möglichst in die bestehende Infrastruktur der Unternehmen integrieren lassen. Oft genügt jedoch die gezielte Erweiterung bestehender Entitäten, um die Funktionalität einer Produktionsanlage an die Bedürfnisse zukünftiger Wertschöpfungsnetzwerke anzupassen (Reiff-Stephan et al. 2015). Im Folgenden sollen verschiedene Lösungen zur Implementierung von industriellen Systemen für die maschinennahe Erfassung energetischer Daten aufgezeigt, verglichen und bewertet werden.

\section{Implementierungskonzepte}

\subsection{Anwendungshintergrund}

Der Bedarf an industriellen Energiemonitoringsystemen soll im Folgenden durch einige Beispiele verdeutlicht werden. Prozessabläufe in den Unternehmen müssen unter betriebswirtschaftlichen Anforderungen in einem zunehmend hohen Maße material- und energieeffizient ausgeführt werden. So kann beispielsweise das energetische Profil eines Prozessschrittes als Entscheidungsfaktor in Produktionsplanungsvorgänge einfließen, wodurch Lastspitzen im fabrikinternen Energienetz vermieden werden (Weinert 2010). Lastspitzen sind hierbei mitunter auf Einschaltprozesse von Maschinen und Anlagen zurückzuführen. Oftmals wird das durch- 


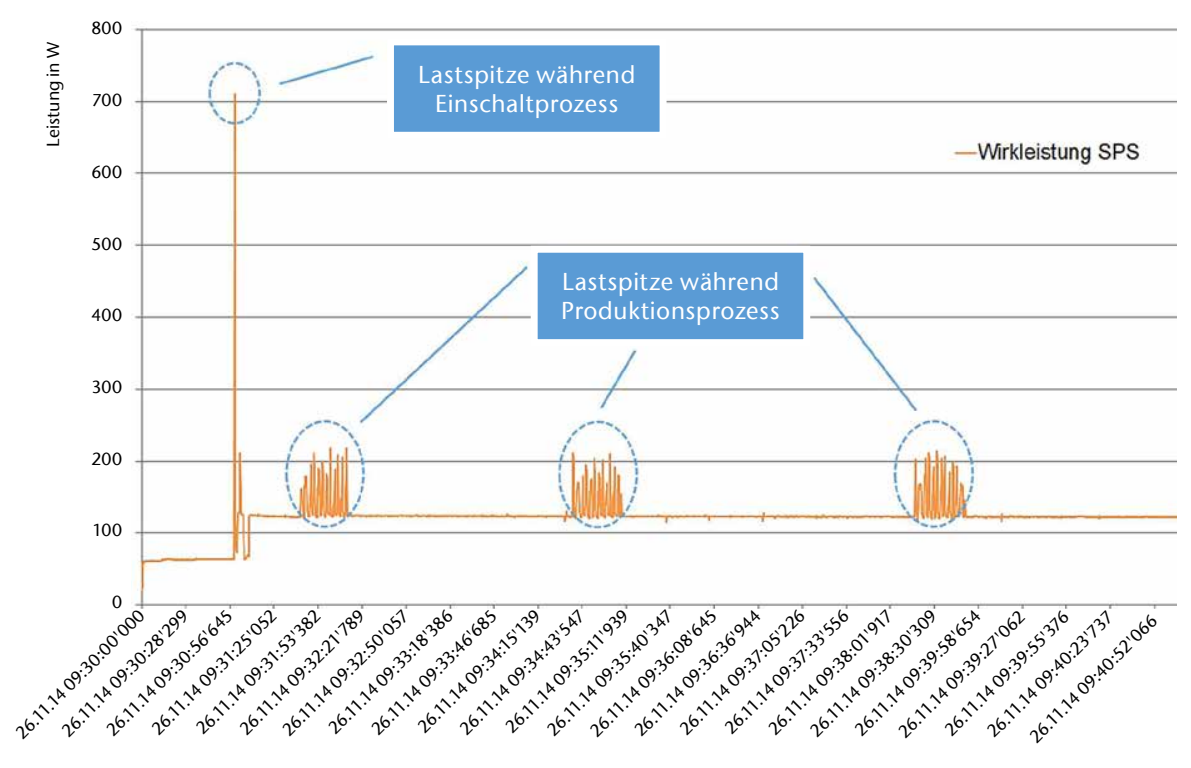

Abb. 1) Lastspitzen während des Einschaltvorgangs einer Werkzeugmaschine

schnittliche Lastprofil des eigentlichen Produktionsprozesses um ein Vielfaches überschritten (Abb. 1). Da die Maximalwerte im Lastprofil die energetischen Bereitstellungskosten direkt beeinflussen, bietet ein intelligentes Lastmanagement hohes Potenzial zur Kostenreduktion (Tönsing 1996).

Des Weiteren kann die genaue Kenntnis über den aktuellen Energiebedarf von Produktionsanlagen, Maschinen und einzelnen Baugruppen Wartungsund Instandhaltungsprozesse unterstützen (Niggemann et al. 2014). So ist zum Beispiel der erhöhte Energiebedarf einer Maschine mitunter ein Indiz für den fortgeschrittenen Verschleiß eines Lagers oder eines Führungselementes. Um diesen Mehrbedarf an Energie zu detektieren, sind jedoch die entsprechenden technischen Entitäten innerhalb der betrieblichen Infrastruktur unabdingbar. In Abb. 2 ist die praktische Anwendung eines derartigen Aufbaus dargestellt.

Ein weiterer Aspekt für den vermehrten Bedarf an technologischen Lösungen für die Erfassung und Verwaltung energetischer Daten ist die zunehmende Nachfrage nach einer Zertifizierung gemäß DIN EN ISO 50001 bzw. DIN EN 16247-1, um beispielsweise an steuerlichen Entlastungen zu partizipieren (Kahlenborn et al. 2012).

\subsection{Anforderungen}

Unter den aufgezeigten Gesichtspunkten besteht ein wesentliches Ziel des Forschungsprojektes MetamoFAB in
Eine wesentliche Herausforderung bei der Implementierung ist die Integration innerhalb bestehender Automatisierungsstrukturen. Entsprechend muss die Kompatibilität zur informationstechnischen Infrastruktur des Unternehmens zum Beispiel durch passende Schnittstellen gewährleistet sein. Des Weiteren ist es erforderlich, dass das System den Anforderungen zukünftiger, durch Industrie 4.0 geprägter, horizontaler wie auch vertikaler Wertschöpfungsnetzwerke gerecht wird (Lipinski et al. 2014). Dies erfordert unter anderem die Unterstützung entsprechender Kommunikationsstandards wie etwa OPC UA oder auch die Sicherstellung der Beherrschbarkeit komplexer Systeme (Niggemann et al. 2014). Neben den geschilderten Anforderungen ist es unabdingbar, dass das technische System den wirtschaftlichen Spezifikationen der geplanten Implementierungsmaßnahmen entspricht. Somit können die Hauptanforderungen an ein Energiemonitoringsystem folgendermaßen klassifiziert werden: kel der hier diskutierten Anwendung, stellt das Sensor- wie auch das übergeordnete Monitoringsystem dar. Primär ist es erforderlich, dass das betrachtete Teilsystem die spezifischen technischen Anforderungen der Messaufgabe erfüllt. Anhand der Messaufgabe werden weiterhin der Messumfang sowie die Mindestanforderungen für Messbereich, Messgenauigkeit, Abtastrate sowie Datenspeicherrate definiert (Müller et al. 2009).

- Sicherstellung der Beherrschbarkeit komplexer Systeme,

- Gewährleistung der Veränderungsfähigkeit,

- Berücksichtigung der Industrie 4.0-Architekturmerkmale,

- Wirtschaftlichkeit sowie

- Erfüllung spezifisch messtechnischer Kriterien.

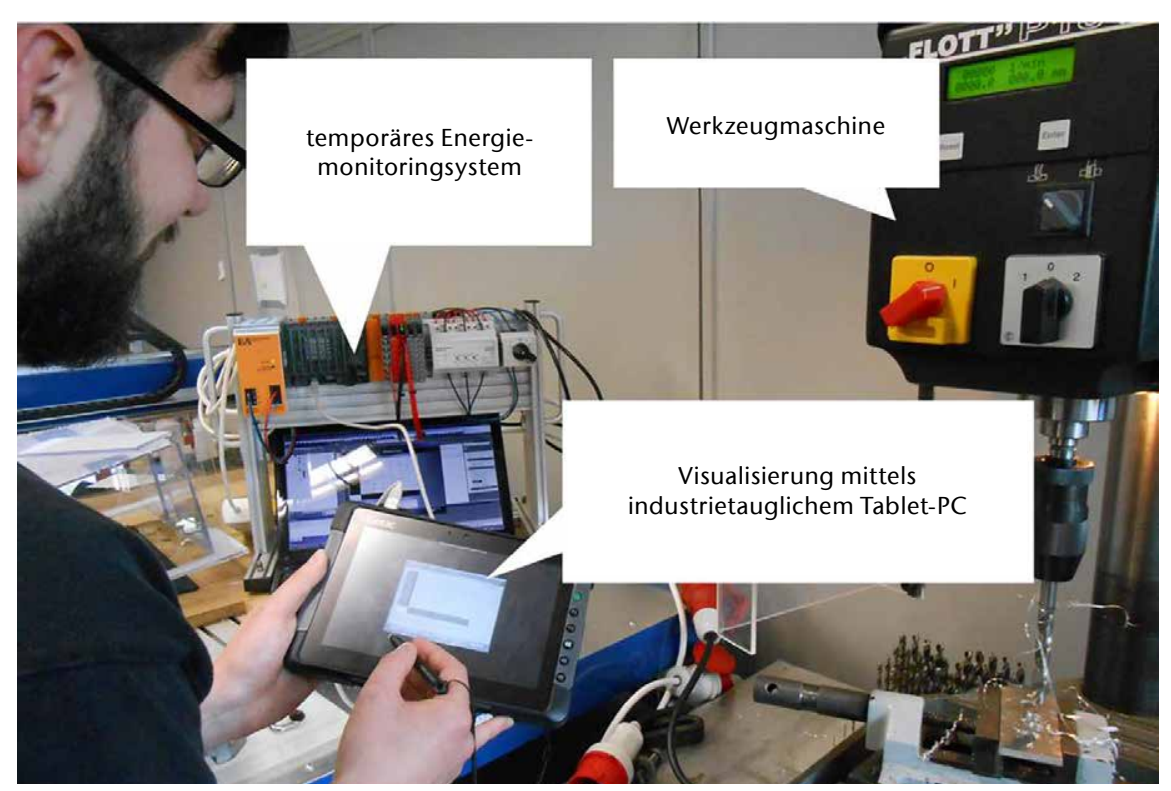

Abb. 2) Erfassung energetischer Daten zur Unterstützung der Wartung einer Werkzeugmaschine 
Tab. 1: Übersicht der untersuchten Implementierungsvarianten

\begin{tabular}{|c|c|}
\hline Benennung & Beschreibung \\
\hline Variante 1 & $\begin{array}{l}\text { Erweiterung einer B\&R SPS (X20) durch } \\
\text { X20 AP } 3131 \text { Energiemessmodul } \\
\text { Datenverwaltung und Visualisierung über } \\
\text { B\&R Automation Studio } \\
\text { Visualisierung der Daten über portables Endgerät }\end{array}$ \\
\hline Variante 2 & $\begin{array}{l}\text { Energiemessgerät Siemens SENTRON PAC } 3200 \\
\text { für Schalttafeleinbau } \\
\text { - Kommunikation über Modbus/TCP oder Ethernet } \\
\text { Datenauswertung mittels Siemens Powermanager }\end{array}$ \\
\hline
\end{tabular}

\begin{tabular}{|l|l}
\hline Variante 3 & $\begin{array}{l}\text { Portabler Messaufbau unter Verwendung } \\
\text { eines Janitza UMG } 604 \text { Moduls }\end{array}$ \\
- Kommunikation über Modbus TCP oder Ethernet \\
Datenauswertung mittels GridVis-5.0.4
\end{tabular}

\section{Variante 4}

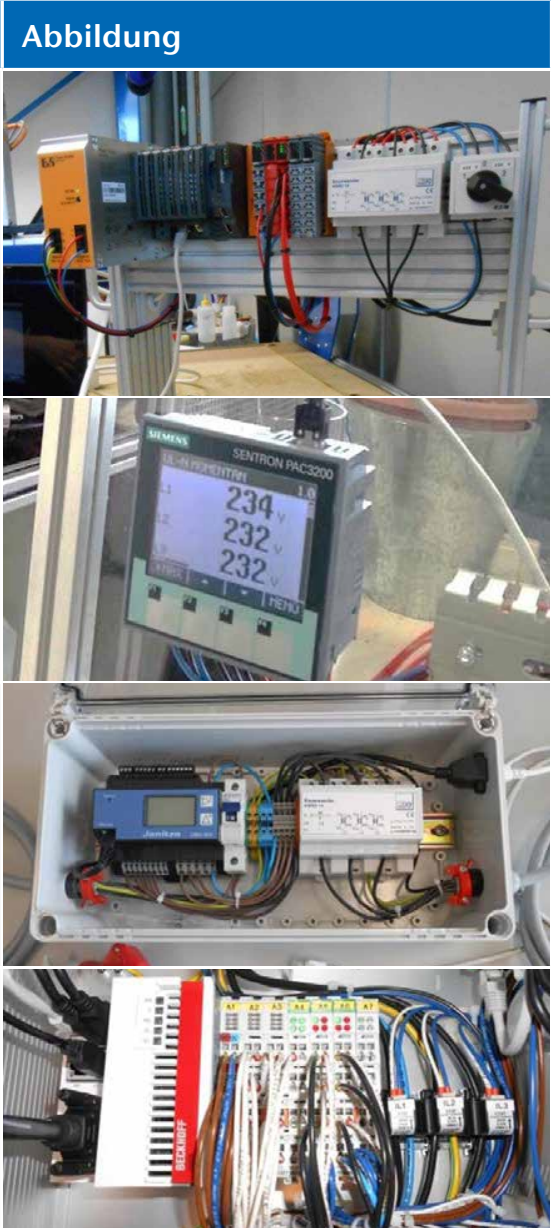

3. Exemplarischer Vergleich anhand marktverfügbarer Komponenten

In Tab. 1 sind vier Implementierungsvarianten aufgeführt, die an der Technischen Hochschule Wildau als Versuchsaufbauten realisiert wurden und einen Auszug der am Markt verfügbaren Lösungen aufzeigen.

Für einen Vergleich der verschiedenen Implementierungsvarianten kommen, abgeleitet aus den zuvor genannten Anforderungen an Energiemonitoringsysteme (siehe 2.2), die in Tab. 2 aufgeführten Bewertungskriterien zur Anwendung. Die für die Versuchsaufbauten verwendeten Systeme wurden auf den Erfüllungsgrad der Bewertungskriterien hin untersucht und nutzwertanalytisch gegenübergestellt. Aufgrund der hohen Anzahl an Bewertungskriterien erfolgt die Gewichtung mit Hilfe von Kriteriengruppen (Kühnapfel 2014). Hierbei werden zunächst die Hauptanforderungsgruppen untereinander gewichtet. Anschließend erfolgt die Gewichtung realisiert werden und mittels geeigneMODBUS/TCP) übergeordneten Steuerungen oder SCADA-Systemen zur al. 2012).

Des Weiteren besteht aber auch die giemessgeräte als Standalone-Lösung. ür die Erstellung von Mess-projekten ler mitunter spezifische Anwenderprogramme zur Verwaltung energetischer Daten angeboten. Als Beispiele seien hier Gridvis (Janitza electronics $\mathrm{GmbH}$ ), Powermanager (Siemens AG) oder Datenverwaltung über grafische Programmiersysteme wie LabVIEW (National Instruments Corporation) umgesetzt werden.

Je nach Komplexität der Messaufgabe und Vernetzungsstruktur kann für die Erfassung energetischer Daten auch auf eine Mischform der zuvor genannten Optionen zurückgegriffen werden. die Entwicklungsumgebung für SPSProgramme des Steuerungsanbieters 
Tab. 2: Übersicht der Bewertungskriterien

Hauptanforderungsgruppe

1 Sicherstellung der Beherrschbarkeit komplexer Systeme

2 Veränderungsfähigkeit

3 Architekturmerkmale Industrie $\mathbf{4 . 0}$

4 Wirtschaftliche Bewertungskriterien

5 Technische Kriterien (ohne direkte Industrie-4.0-Relevanz)
Bewertungskriterium

1.1 Supportfaktor

1.2 Kompetenzfaktor

2.1 Abwärtskompatibilität

2.2 Erweiterbarkeit

2.3 Adapterorientierte Funktionalität

3.1 Durchgängigkeit der Daten entlang der Prozesskette

3.2 Einsatz von Standards in der Kommunikation

3.3 Gleichzeitige Unterstützung von zentraler und dezentraler Intelligenz

3.4 Interoperabilität

4.1 Anschaffungskosten Gesamtsystem

4.2 Anschaffungskosten Ergänzungssystem

4.3 Zeitaufwand für Implementierung

5.1 Messgenauigkeit

5.2 Mean Time Between Failures (MTBF)

5.3 Benötigter Bauraum

5.4 Updatezeit (Messwert)

5.5 Auflösung

Energieeffizienz (Scheinleistungsaufnahme) der Kriterien innerhalb der einzelnen Hauptanforderungsgruppen.

Auf die Bewertungskriterien der Hauptanforderungsgruppe 1 soll im Folgenden exemplarisch noch näher eingegangen werden. Für die Bewer-

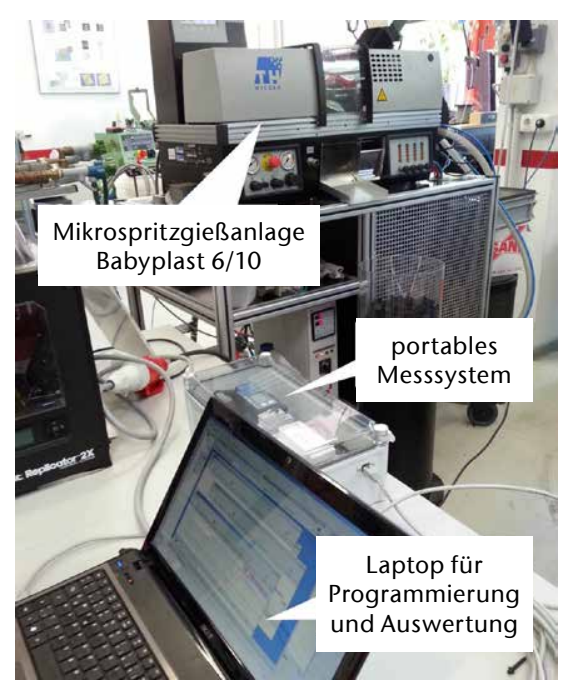

Abb. 3) Versuchsaufbau zur Erfassung energetischer Daten eines Spritzgussprozesses tung der Beherrschbarkeit komplexer Systeme sind insbesondere Nutzungsfaktoren von ausschlaggebender Bedeutung.

So dient etwa der "Supportfaktor" (1.1) als Bewertungsgröße für die Beurteilung, inwieweit der Anwender durch den Hersteller bei Implementierung und Betrieb des Systems Unterstützung erfährt. In die Bewertung fließen hierbei die Qualität sowie die Verfügbarkeit von Dokumenten wie Bedienungsanleitungen oder Hilfedateien ein. Des Weiteren wird die Kundenbetreuung bewertet.

Hingegen dient der „Kompetenzfaktor“ (1.2) zur Beurteilung, ob vom Anwender/Bediener spezielle Fähigkeiten bzw. Kompetenzen zur Benutzung des Systems verlangt werden. Dies wurde durch die Erstellung eines Referenzprojekts zur Messung mehrerer definierter energetischer Daten am Beispiel eines Spritzgussprozesses untersucht.

Neben den zuvor erwähnten Nutzungsfaktoren ist die eigentliche Funk- tionserfüllung, also die Aufnahme der Energie- und Verbrauchsdaten, ein wesentlicher Aspekt bei der Bewertung der Energiemonitoringsysteme. Diese wurde durch die praktischen Versuche geprüft. In Abb. 3 ist einer der exemplarischen Versuchsaufbauten an einer Mikrospritzgießanlage Babyplast 6/10 dargestellt.

In Abb. 4 ist das aufgenommene Lastprofil der Wirkleistung für einen Spritzgussprozess von hundert Zyklen ersichtlich. Hierbei handelt es sich um eine mit der Reportfunktion von Janitza GridVis erstellte Grafik. Die Zeit für das Einspritzen des Kunststoffes beträgt während des Versuchs eine Sekunde je Schuss. Pro Schuss erfolgt die Fertigung von zwei Einzelstücken. Über den Versuchszeitraum wurde die Kühlzeit von 15 Sekunden über 7,5 Sekunden bis auf 3,5 Sekunden schrittweise reduziert. Anhand der Grafik ist ersichtlich, dass es über den gesamten Aufzeichnungszeitraum aufgrund der gesteuerten Prozessführung zu deutlichen 


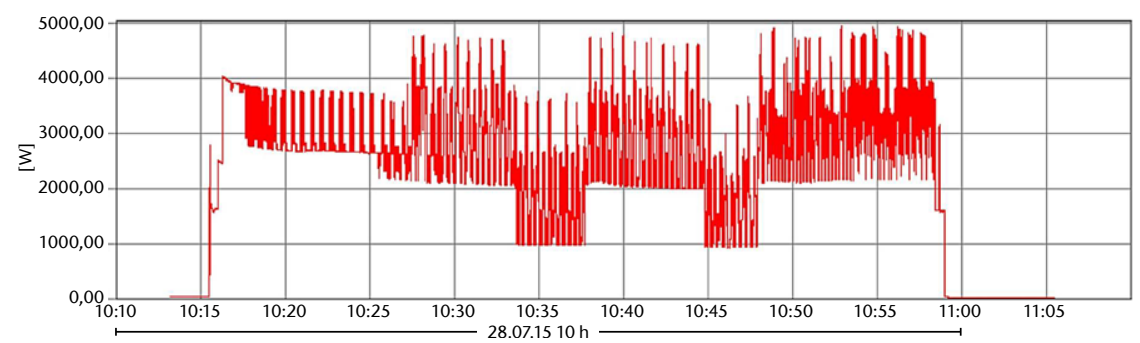

Abb. 4) Wirkleistungsprofil der Leiter $L 1$ bis $L 3$ für einen Spritzgussprozess als Auszug eines Janitza GridVis Reports. Untersucht wurden in der Aufschaltung der Anlage die drei Stromleiter der Spritzgießanlage. Diese dienen jeweils der separaten Energieversorgung der Hauptaggregate Heizung (L1), Hydraulik (L2) und Kühlung (L3). Durch Betrachtung der leitungsbezogenen Effektivstromkurven (Abb. 5) kann den jeweiligen Aggregaten der entsprechende Anteil am Lastprofil zugeordnet werden.

Effektivstrom L1 (Heizung)

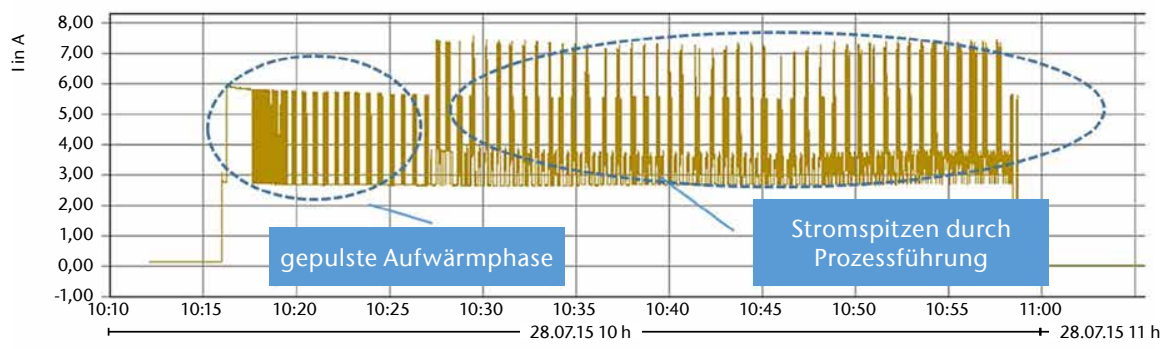

Effektivstrom L2 (Hydraulik)

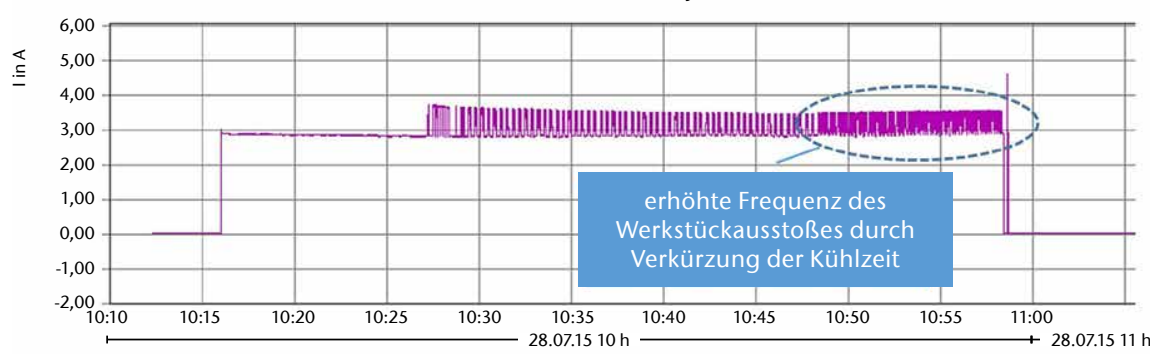

Effektivstrom L3 (Kühlung)

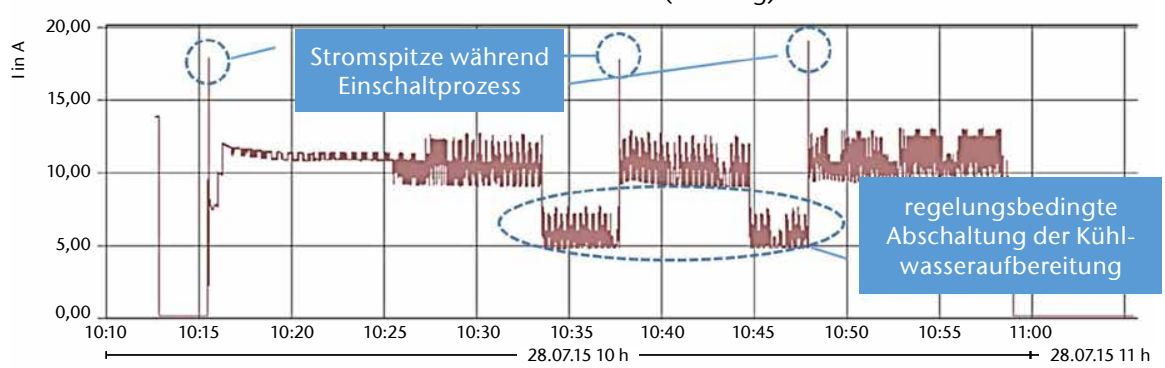

Abb. 5) Effektivströme der Leiter L1 bis L3 für einen Spritzgussprozess als Auszug eines Janitza GridVis Reports

Schwankungen im Lastprofil kommt. Es ist zu erkennen, dass für die Abführung der Prozesswärme ein Großteil elektrische Energie zur Verfügung zu stellen ist. Die Stromspitzen im Leiter L3 erreichen in den verschiedenen Einschaltphasen einen Wert von bis zu 19 A. Ebenso liegt das Stromgrundniveau mit 11 A deutlich oberhalb dessen der anderen Stromleitungen. Ebenfalls ist das Absinken der Grundlast durch die reglungsbedingte zeitweilige Abschaltung der giedaten bereitstellen. Die Leistungsprofile können entsprechend exakt aufgenommen und die Daten in die Datenerfassungssysteme überführt werden. Der dafür erforderliche Aufwand findet Berücksichtigung in der gesamtheitlichen, nutzwertanalytischen Bewertung. Je nach Anforderungsprofil des Unternehmens variiert die Relevanz der hinzugezogenen Kriterien und es bedarf einer Gewichtung. Für die Untersuchung im Rahmen der Studie erfolgte die Gewichtung der Kriterien für ein Unternehmen mit KMU-Struktur. Es wurde hierzu insbesondere ein hoher Stellenwert auf den Support- und Kompetenzfaktor gelegt. Dies ist damit zu begründen, dass KMUs oft sehr spezifisch geschulte Experten in ihren Reihen aufweisen und nicht im gleichen Maße auf ein so breit gefächertes Portfolio an Kompetenzträgern zurückgreifen können wie Großunternehmen.

Die Ergebnisse der nutzwertanalytischen Gegenüberstellung der verschiedenen Implementierungsvarianten sind in Abb. 6 dargestellt. Es ist festzustellen, dass die Implementierungsvariante 2 mit einem Nutzwert von $78 \%$ die größte Übereinstimmung mit dem Anforderungsprofil des exemplarischen Nutzers aufweist. Auf einzelne Ergebnisse der Untersuchung soll im Folgenden näher eingegangen werden.

Innerhalb der Untersuchungen hat sich gezeigt, dass die Erstellung des Referenzprojekts und die anschließenden Messungen mit allen Systemen mit hinreichender Genauigkeit durchführbar waren. Es ist jedoch festzustellen, dass gerade für die integrativen Lösungen für SPS und IPCs ein Grundmaß an Praxiserfahrung im Umgang mit der jeweiligen Programmierumgebung den Zeitaufwand für die Programmerstellung deutlich verringert. Es ist davon auszugehen, dass Unternehmen mit entsprechenden technischen Entitäten und Erfahrungsträgern derartige Lösungen schnell implementieren können. Im Vergleich hierzu hat sich gezeigt, dass Standalone-Lösungen in Kombination mit den spezifischen Anwenderprogrammen geringere Anforderungen an das Kompetenzprofil des Anwenders stellen. Die Programmierung bzw. die Konfiguration war hier auch bei wenig Pra-
Es kann festgestellt werden, dass alle vorgestellten Varianten die Messgenauigkeiten zur Erfassung der Ener-
Kühlwasseraufbereitung eindeutig liegt das Strom Vergleich da energetische Versorgung der hydraulischen Aktuatoren bei nur etwa $3 \mathrm{~A}$ und wird durch die Prozessführung nur um etwa $20 \%$ erhöht.

\section{Auswertung}




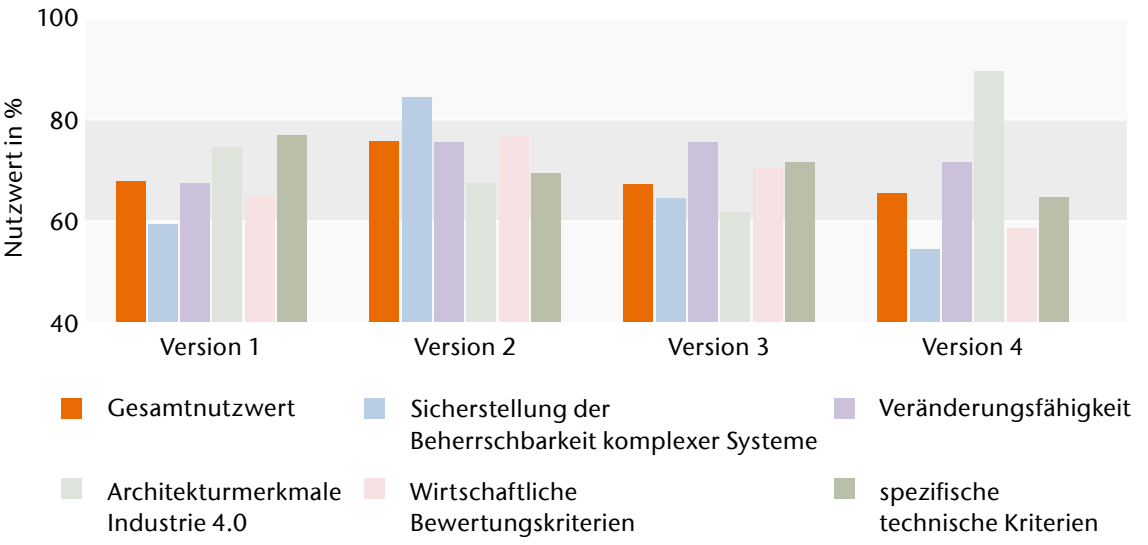

Abb. 6) Gegenüberstellung der Nutzwerte

xiserfahrung mit einem vertretbaren Zeitaufwand zu realisieren. Bei einer Entscheidung für eine bestimmte Implementierungsvariante müssen demnach, je nach Ausgangssituation, die spezifischen Fähigkeiten der menschlichen Entitäten eines Unternehmens besichtigt, werden, um die Beherrschbarkeit komplexer Systeme positiv zu beeinflussen.

Ein weiterer Aspekt der aus der Untersuchung der verschiedenen Varianten hervorgeht, ist die differenzierte Betrachtungsweise der Implementierungskosten. Ohne vorhergehende Analyse der bestehenden Infrastruktur und genaue Definition der technischen Anforderungen können die Kosten für ein Energiemonitoringsystem unverhältnismäßig hoch ausfallen. Sind zum Beispiel die technischen Spezifikationen einer integrativen Lösung für eine bestimmte Messaufgabe ausreichend, belaufen sich die Implementierungskosten im Wesentlichen auf die Anschaffungskosten der Messkarte. Dies setzt natürlich voraus, dass die technische Infrastruktur des Unternehmens über entsprechende IPCs bzW. SPS und die passende Softwareumgebung verfügt. In diesem Fall ist mit den im Test untersuchten Messkarten ein Energiemonitoringsystem mit Hardwareinvestitionskosten von $200 €$ bis $300 € \mathrm{zu}$ realisieren. Kann hingegen nicht auf integrative Lösungen angeknüpft werden, verschiebt sich der Kostenvorteil in Richtung der Standalone-Systeme.

Es ist festzustellen, dass die Auswahl einer geeigneten Implementierungsvariante für ein Energiemonitoringsystem stark von den Ausgangsbedingungen des jeweiligen Unternehmens abhängig ist. Somit kann keine allgemeingültige Aussage über den Grad der Eignung einer Variante für einen breiten Kreis von Unternehmen getroffen werden. Hieraus leitet sich der Bedarf nach Modellen zur individuellen Bewertung und Auswahl von Implementierungsstrategien ab. Im Rahmen des Forschungsprojektes MetamoFAB (BMBF 2015) erarbeitet die iMEP-Forschungsgruppe „Automatisierungstechnik" der Technischen Hochschule Wildau als assoziierter Partner ein derartiges Modell. Diese soll den Unternehmen dabei helfen, Zielgrößen für technische Entitäten zu definieren und über Analyse- und Bewertungsprozesse eine geeignete Implementierungsstrategie abzuleiten.

\section{Fazit}

Bezogen auf die untersuchten Systemvarianten kann aufgrund der Vielfalt der Lösungsansätze keine abschließende Aussage getroffen werden, ob beispielsweise System $A$ gegenüber System B zu bevorzugen ist. Vielmehr zeigt der Vergleich auf, dass für den jeweiligen Anwendungsfall das geeignetste System zu identifizieren ist. Mit den gefundenen Lösungen wird es möglich, Managementsysteme zu entwickeln und zu betreiben, um den Energiebedarf von Anlagen und Maschinen entsprechend der Lastbereitstellung effizient zu nutzen. Es müssen hierfür jedoch kundenindividuelle Lösungen gefunden werden, da eine Standardisierung in Produkten und Dienstleistung noch nicht allgemein bereitgestellt wird. Im Rahmen der Forschungsarbeiten an der Hochschule wird an einem Implementierungs- modell zum Einsatz informationstechnischer Unterstützungswerkzeuge für schnelle Produktionsprozesse gearbeitet. Die hier vorgestellten Ansätze gehen als Lösungsmöglichkeiten in die ganzheitliche Betrachtung mit ein.

\section{Förderhinweis}

Das Forschungs- und Entwicklungsprojekt „Metamorphose zur intelligenten und vernetzten Fabrik“ (MetamoFAB) wird mit Mitteln des Bundesministeriums für Bildung und Forschung (BMBF) im Rahmenkonzept „Forschung für die Produktion von morgen" (Förderkennzeichen 02PJ4040 ff) gefördert und vom Projektträger Karlsruhe (PTKA) betreut. Die Verantwortung für den Inhalt dieser Veröffentlichung liegt bei den Autoren.

\section{稀 Bundesministerium \\ für Bildung und Forschung \\ PTKA \\ Projektträger Karlsruhe Karlssuher Institut für Technologie}

\section{LITERATUR}

Bauer K, Diegner B, Diemer J, Dorst W, Ferber S, Glatz R, Hellinger A, Herfs W, Horstmann M, Kaufmann T, Kurz C, Löwen U, Stumpf V (2013) Deutschlands Zukunft als Produktionsstandort sichern. Umsetzungsempfehlungen für das Zukunftsprojekt Industrie 4.0: Abschlussbericht des Arbeitskreises Industrie 4.0. a der

BMBF (2015) Industrie 4.0. Innovation für die Produktion von morgen. https://www.bmbf.de/pub/ Industrie_4.0.pdf. Accessed 14 Sep 2015

Dorst W, Glohr C, Hahn T, Knafla F, Loewen U, Rosen R, Schiemann T, Vollmar F, Winterhalter C (2015) Umsetzungsstrategie Industrie 4.0: Ergebnisbericht der Plattform Industrie 4.0. BITKOM e. V., VDMA e. V., ZWEI e. V., Frankfurt/Main

Kahlenborn W, Kabisch S, Klein J, Richter I, Schürman S (2012) Energiemanagementsysteme in der Praxis. ISO 50001: Leitfaden für Unternehmen und Organisationen. Bundesministerium für Umwelt, Naturschutz und Reaktorsicherheit; Umweltbundesamt, Berlin/Dessau

Krüger J, Parthey H, Wink R (eds) (2015) Wissenschaft und Innovation. Wissenschaftsforschung Jahrbuch 2014. Wissenschaftlicher Verlag Berlin WVB, Berlin. ISBN: $978-$ 3-86573-841-7

Kühnapfel JB (2014) Nutzwertanalysen in Marketing und Vertrieb. Springer Gabler, Wiesbaden. ISBN: 978-3-65805508-0. doi: 10.1007/978-3-658-05509-7

Lipinski R von, Richter M, Reiff-Stephan J (2014) Intelligent sensor systems for self-optimizing production chains. In: Proc 1st Int Conf Exhib Future RFID Technol (FutureRFID 2014), 6-7 Nov 2014, Eger. Eszterházy Ká(FutureRFID 2014), 6-7 Nov 2014, Eger. Eszterházy Ká23-0, pp 115-125. doi: 10.17048/FutureRFID.1.2014.115 
Maier A, Pethig F, Vodencarevic A, Schetinin N, Niggemann O, Kleine Büning H (2012) Analyse und Visualisierung des Energieverbrauchs in Produktionsanlagen. In: Adolphs P (ed) Automation 2012. VDI-Verlag, Düsseldorf, ISBN: 978-3-18-092171-6

Müller E, Engelmann J, Löffler T, Strauch J (2009) Energieeffiziente Fabriken planen und betreiben. Springer, Berlin/Heidelberg. ISBN: 978-3-642-31945-7. doi: 10.1007/978-3-540-89644-9

Niggemann O, Jasperneite J, Vodencarevic A (2014) Konzepte und Anwendungsfälle für die intelligente $\mathrm{Fa}$ Konzepte und Anwendungsfälle für die intelligente Fa-
brik. In: Bauernhansl T, ten Hompel M, Vogel-Heuser B brik. In: Bauernhansl T, ten Hompel M, Vogel-Heuser B
(eds) Industrie 4.0 in Produktion, Automatisierung und (eds) Industrie 4.0 in Produktion, Automatisierung und
Logistik. Anwendung - Technologien - Migration. Springer Vieweg, Wiesbaden, ISBN: 978-3-658-04681-1, pp 173-190. doi: 10.1007/978-3-658-04682-8_9

Reiff-Stephan J, Richter M, Lipinski R von (2015) Intelligente Sensorsysteme für selbstoptimierende Produktionsketten. In: Tagungsband zur 12. AALE-Fachkonferenz. Deutscher Industrieverlag, München, ISBN: 978-3-83567270-3, pp 245-354

Tönsing E (1996) Energiekostenreduzierung durch betriebliches Energiemanagement. Fachartikel im Rahmen der Initiative „Energie effizient nutzen - Schwerpunkt Strom“. Wirtschaftsministerium Baden-Württemberg; Fraunhofer-Institut für System- und Innovationsforschung, Stuttgart/Karlsruhe

Weinert N (2010) Vorgehensweise für Planung und Betrieb energieeffizienter Produktionssysteme. Berichte aus dem Produktionstechnischen Zentrum Berlin. Fraunhofer-Verlag, Stuttgart. ISBN: 978-3-8396-0173-0

Weinert N (2015) Verbundprojekt MetamoFAB - Metamorphose zur intelligenten und vernetzten Fabrik. 3. Klausurtagung Automatisierungstechnik: Automatisierte Produktionsprozesse der Zukunft, Technische Hochschule Wildau, 23 Apr 2015. http://www.th-wildau. de/fileadmin/dokumente/studiengaenge/automatisierungstechnik/dokumente/04_2015-04-23_Weinert MetamoFAB_Cesamt\%C3\%BCberblick_final.pdf. Accessed 27 Oct 2015

\section{AUTOREN}

René von Lipinski, M. Eng

Prof. Dr.-Ing. Jörg Reiff-Stephan

Technische Hochschule Wildau

Institut für Entwicklung, Material und Produktion (iMEP)

Forschungsgruppe: Automatisierungstechnik

Dipl.-Ing. Dirk Buße

Geschäftsführer Budatec $\mathrm{GmbH}$, Berlin

E-Mail für Korrespondenz:

rene.von_lipinski@th-wildau.de 